\title{
Choledochal Cyst: Their clinical presentation, diagnosis and treatment in central Nepal: A retrospective study
}

Sujit Kumar, B.N Patowary,Aditya Jalan

Department of Surgery, College of Medical Sciences-Teaching hospital,Bharatpur, Chitwan , Nepal

Correspondence : Dr Sujit Kumar, Associate professor, Department of Surgery, College of Medical sciencesTeaching Hospital ,Bharatpur, Chitwan, Nepal

email drsujit1755@gmail.com

\begin{abstract}
Background: Choledochal cyst is a rare congenital malformation involving the cystic dilatation of intrahepatic or extra-hepatic or both bile duct .The estimated incidence is one in 1000 live birth in Asian population with female to male ratio $3: 1$.
\end{abstract}

Objectives:To study the clinical presentations, diagnostic modalities, treatment and outcome of choledochal cyst in College of Medical Sciences, Bharatpur, Nepal

Methods:A retrospective review of the records of all the patients who were diagnosed as choledochal cyst and underwent medical or operative intervention in our hospital in the period of January 2013- January 2015.

Results:We have analyzed ten cases of choledochal cyst. We found most common age group of presentation of choledochal cyst was 6-10 yr with female preponderance (70\%). Most common presenting symptom was pain abdomen (100\%) followed by jaundice (50\%). However classical triad of abdominal pain, jaundice, and an abdominal mass was not seen in any of the cases. Ultrasonography was found to be $100 \%$ accurate in diagnosis of choledochal cyst. Todani type I cyst was the most common type. Nine out of ten cases underwent complete cyst excision with cholecystectomy and Roux-en-Y hepaticojejunostomy without any major complication. Malignancy was not seen in any patients.

Conclusion: Choledochal cyst is a rare disease. Most common age group of presentation is 6-10 yrs. Pain abdomen is the most common clinical presentation. Ultrasonography is usually the first examination and is very sensitive for diagnosis of choledochal cyst. Complete cyst excision, cholecystectomy and internal drainage is the treatment of choice.

Keywords: Choledochal cyst, ultrasonography, roux-en-y hepaticojejunostomy 